\title{
Nonequilibrium mesoscopic transport: a genealogy
}

\author{
Mukunda P. Das ${ }^{1}$ and Frederick Green ${ }^{2}$ \\ ${ }^{1}$ Department of Theoretical Physics, Research School of Physics and \\ Engineering, The Australian National University, Canberra, ACT 0200, \\ Australia. \\ ${ }^{2}$ School of Physics, The University of New South Wales, Sydney, NSW 2052, \\ Australia.
}

\begin{abstract}
Models of nonequilibrium quantum transport underpin all modern electronic devices, from the largest scales to the smallest. Past simplifications such as coarse graining and bulk self-averaging served well to understand electronic materials. Such particular notions become inapplicable at mesoscopic dimensions, edging towards the truly quantum regime. Nevertheless a unifying thread continues to run through transport physics, animating the design of small-scale electronic technology: microscopic conservation and nonequilibrium dissipation. These fundamentals are inherent in quantum transport and gain even greater and more explicit experimental meaning in the passage to atomic-sized devices. We review their genesis, their theoretical context, and their governing role in the electronic response of meso- and nanoscopic systems.
\end{abstract}

\section{Introduction}

As soon as it became possible to fabricate conducting devices at scales comparable to the scattering mean free paths of the underlying material, experiments began to reveal some remarkable departures from bulk electrical-response behaviour. Among the earliest notable phenomena were universal conductance fluctuations [1, followed by the first measurements of conductance quantization itself, probed as a function of carrier density in the device [2, 3].

These novel effects highlighted the inadequacy of standard theoretical techniques, which had proved themselves extraordinarily effective in the rapid development of solid-state electronics right up to the 1980s. Typical of these older procedures is impurity-site averaging for conductance calculations in the bulk; relying as it does on an assumption of homogeneous randomness, spatial averaging breaks down when the conducting channel of an actual sample is too small to hold more than a few impurities, if any at all.

A second, more basic, instance is the limit to the applicability of Bloch's theorem. Standard band-structure analysis fails in the absence of lattice regularity involving, again, an assumption of large-scale uniformity. Few of the new types of structure are defined by this level of periodicity.

Of prime significance to mesoscopic transport is the openness and intimacy of contact between the nominally active region and its macroscopic environment. These complements - the mesoscopic structure and its surroundings - are no longer subject to conventional approximations in which one part of the complete system couples only weakly, or quasi-independently, to the other. The physics of the interfaces is 
conceptually inseparable from the core device, and constitutes the major issue. Even the simplest of questions, such as how to define the very working "length" of such structures, can turn into a subtle problem.

The literature is replete with texts and reviews, treating in detail every approach to mesoscopics that now enjoys currency [4]- 18]. In the overview to follow we aim not so much to retrace this well-trodden ground as to probe (even at a risk of pedantry) the fundamental underpinnings that today's mesoscopic transport theories may wish to claim, rightly, to be integral to themselves. For up-to-date technicalities we direct readers to representative treatments; those cited above are just a sample.

In meeting our goal we will cover the main conceptual points that must underwrite successful mesoscopic approaches. This brings us to the central question. Precisely what are the minimal requirements on practical methods, as imposed by the underlying principles of quantum physics? The answer hangs upon the real nature and role of multi-particle dynamics.

Our paper is set up as follows. In Section 2 we recall standard methods of microscopic analysis in light of the new problems set by carrier physics on very small scales. The methods are: (1) the quantum Boltzmann equation; (2) the Kubo formula; (3) field-theory based methods (Kadanoff-Baym, Keldysh); and (4) the Landauer formula. Despite procedural differences, the methods aim to share a common basis in the dominance of the conservation laws. Together with the many-body nature of transport, that is the unifying thread.

In Section 3 we focus on models of conductance quantization and the treatment of dissipation, conditioned by the intimate connection with fluctuations and hence with thermodynamic stability in any normal conduction process. We show that exclusively linear models of mesoscopic response are not guaranteed to capture, uniquely, the physics of stable conduction. For, a system's stability is determined by its essentially nonlinear dynamics; an aspect which has hardly been investigated in mesoscopics and which we explore below through a simple model. Finally we summarize this overview in Sec. 4.

\section{Approaches to Quantum Transport}

\subsection{Quantum Boltzmann Equation}

We start with some primary kinetic ideas, reaching back to Maxwell and Boltzmann. Boltzmann's transport equation [19, 20, 21, 22] provides the opportunity to highlight concepts that have grown to their broadest significance in the quantum realm.

A quantum extension to the Boltzmann equation, first introduced by Uehling and Uhlenbeck [19, describes metallic carrier dynamics at scales of length and time long compared to the Fermi wavelength and frequency. A related transport equation for the degenerate, interacting-electron liquid was formulated by Landau and Silin 23.

The subject of the quantum Boltzmann equation (QBE) is the mean distribution of carriers as it changes over time $t$ within the single-particle configuration space for position and wavevector $(\mathbf{r}, \mathbf{k})$ :

$$
\left[\frac{\partial}{\partial t}+\mathbf{v}_{\mathbf{k}} \cdot \frac{\partial}{\partial \mathbf{r}}+\frac{\mathbf{F}(\mathbf{r}, t)}{\hbar} \cdot \frac{\partial}{\partial \mathbf{k}}\right] f_{\mathbf{k}}(\mathbf{r}, t)=-\left[\frac{\partial f}{\partial t}\right]_{\text {coll }}(\mathbf{k} ; \mathbf{r}, t) .
$$

With group velocity $\mathbf{v}_{\mathbf{k}}$ in the carrier band and the force field $\mathbf{F}(\mathbf{r}, t)$ acting on each carrier, the left-hand side of the equation gives the convective derivative: the intrinsic 
or proper rate of change of the mean particle distribution $f_{\mathbf{k}}(\mathbf{r}, t)$ in a frame co-moving with the semiclassical flow. The right-hand quantity in Equation (10) accounts for the loss (net outflow from the state labelled $\mathbf{k}$ ) via the microscopic scattering processes active in the system.

The quantum Boltzmann equation provided the kinetic template for successive, more general nonequilibrium theories. The key to its enduring success lies in Boltzmann's original formulation of the collision term and its method of construction. But before anything else can be said about collision physics, conservation must be set in place as the prime, overarching physical constraint.

The unconditional requirement for conservation emerges when the momentum dependence of the one-body distribution is integrated over, to obtain the local number density and flux; respectively,

$$
\rho(\mathbf{r}, t)=\Omega^{-1} \sum_{\mathbf{k}} f_{\mathbf{k}}(\mathbf{r}, t) \text { and } \mathbf{j}(\mathbf{r}, t)=\Omega^{-1} \sum_{\mathbf{k}} \mathbf{v}_{\mathbf{k}} f_{\mathbf{k}}(\mathbf{r}, t)
$$

with $\Omega$ a volume cell, centred at $\mathbf{r}$, over which the band structure is (locally) defined. Applying the trace operation of Eq. (2) to each side of the QBE produces

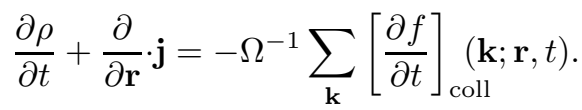

In a system closed to particle exchange with external reservoirs (as for a working electrical circuit) the continuity equation, Eq. (3) above, must be source-free. The carrier number density is microscopically conserved if, and only if, the trace sum on the right-hand side of the relation vanishes identically to lead to the equation of continuity.

Why is this the crucial fact, and why even recall such an "elementary" given? First, it is crucial to each and every model because, otherwise, charge could appear and disappear arbitrarily and beyond the model's control. Nor does conservation come cheaply, in terms of intellectual effort. Second, no matter how cosmetically attractive its other features might be, a scheme that was unable to account for its microscopic conservation properties would face a serious credibility problem, practically as well as formally.

The primacy of conservation and continuity leaves two options for progress. To begin with, microscopic analysis of the Boltzmann collision term will guarantee its vanishing trace. Let us apply the Uehling-Uhlenbeck construction of the scattering rate to Fermi particles [19, 21]. The term corresponding to collisions of, say, individual electrons with a set of external scattering agents (the particle-particle collision integral is more complex, but analogously structured) is

$$
\begin{aligned}
{\left[\frac{\partial f}{\partial t}\right]_{\text {coll }} \equiv \sum_{\mathbf{k}^{\prime}} } & {\left[\left(1-f_{\mathbf{k}^{\prime}}(\mathbf{r}, t)\right) Q_{\mathbf{k}^{\prime} \leftarrow \mathbf{k}} f_{\mathbf{k}}(\mathbf{r}, t)\right.} \\
- & \left.\left(1-f_{\mathbf{k}}(\mathbf{r}, t)\right) Q_{\mathbf{k} \leftarrow \mathbf{k}^{\prime}} f_{\mathbf{k}^{\prime}}(\mathbf{r}, t)\right],
\end{aligned}
$$

where the form of the transition rate $Q_{\mathbf{k}^{\prime} \leftarrow \mathbf{k}}$ carries the collision physics.

Equation (4) differs from the classical through its explicit allowance for Pauli blocking of the outgoing scattering states, a quintessentially nonclassical effect and the most overt of the quantum phenomena entering into normal metallic transport. The leading contribution to the right-hand side sum sends a particle out of state $\mathbf{k}$ 
to state $\mathbf{k}^{\prime}$. Its counter-term accounts for the inverse process whereby a new carrier enters state $\mathbf{k}$ out of $\mathbf{k}^{\prime}$.

The scattering rate is local in space and time, consistent with the long-wavelength time-local (Markov) structure of the QBE's left-hand side. Other than Pauli exclusion, all the short-range correlations or coherences in time and space, on the scale of the Fermi frequency and wavelength, are subsumed in $Q_{\mathbf{k}^{\prime} \leftarrow \mathbf{k}}$. These "intra-collisional" quantum effects do not directly influence the QBE's kinetic shape.

The collision term on the right of Eq. (4) is traceless. It satisfies conservation automatically but, in full form, it can pose technical difficulties to an effective solution. The level of practical effort worth spending on it naturally depends on the fidelity we require in the answer. Even with large computer resources the competing demands of inhomogeneity, realistic and complex band structures, and so on may justify a simplified approach to the scattering properties of the QBE as such.

At this point the alternative option offers itself. We can try for a much simpler Ansatz for the collision integral. If the new trace structure fails to vanish identically, however, conservation may have to be imposed as an additional constraint, no longer given for free.

An early and still popular collision approximation is the Drude model, shown here in its most basic form:

$$
\begin{aligned}
{\left[\frac{\partial f}{\partial t}\right]_{\text {coll }} \equiv } & \frac{1}{\tau_{\mathrm{el}}}\left(f_{\mathbf{k}}(\mathbf{r}, t)-\left\langle f_{\mathbf{k}^{\prime}}(\mathbf{r}, t)\right\rangle_{E_{\mathbf{k}^{\prime}}=E_{\mathbf{k}}}\right) \\
& +\frac{1}{\tau_{\text {in }}}\left(f_{\mathbf{k}}(\mathbf{r}, t)-f_{\mathbf{k}}^{\mathrm{eq}}(\mathbf{r}, t)\right) .
\end{aligned}
$$

The elastic and inelastic collision times $\tau_{\mathrm{el}}$ and $\tau_{\text {in }}$ reflect, in a coarse-grained way, the more complex physics of the original scattering prescription, Eq. (44). The Drude collision times can depend on the single-particle energy $E_{\mathbf{k}}$; here we choose $\tau_{\mathrm{el} \text {;in }}$ as constants, purely to streamline the discussion.

The equilibrium distribution $f^{\mathrm{eq}}$ within the inelastic term, second on the right of Eq. (5), is the usual Fermi-Dirac function in terms of the thermal energy $k_{\mathrm{B}} T$ and global chemical potential $\mu$, as fixed by the environment:

$$
f_{\mathbf{k}}^{\mathrm{eq}}(\mathbf{r}, t) \equiv\left(1+\exp \left(\left(E_{\mathbf{k}}+u(\mathbf{r}, t)-\mu\right) / k_{\mathrm{B}} T\right)\right)^{-1}
$$

where $u(\mathbf{r}, t)$ is an effective local potential, to be determined. In the elastic contribution, first on the right of Eq. (5), the expectation $\left\langle f_{\mathbf{k}^{\prime}}\right\rangle_{E_{\mathbf{k}^{\prime}}=E_{\mathbf{k}}}$ averages $f$ over all possible wavevector orientations, on the assumption that an elastic collision randomizes the exit wavevector completely, instantaneously, and locally but without energy exchange between carrier and scatterer.

The elastic Drude term gives an instance where the zero-trace property of the exact collision integral is structurally preserved. Unfortunately, its inelastic partner does not have this advantage and needs special attention.

As with the elastic component, the assumption behind the inelastic Drude term is that the collision instantaneously and totally randomizes the perturbed distribution, resetting it to (local) equilibrium. In the process the mean energy density of the carriers is locally changed, but the particle density cannot change. This is precisely because all scattering processes are conceived as strictly localized in real space. The need for conservation forecloses any option for a discontinuous change in local carrier number. 
A conserving procedure for the inelastic Drude term was originally proposed by Greene et al. 24] and independently by Mermin 25. The local potential $u(\mathbf{r}, t)$ is determined by demanding that the trace of the inelastic term should be zero for all $(\mathbf{r}, t)$. For the actual system density $\rho(\mathbf{r}, t)$ this requires that

$$
\Omega^{-1} \sum_{\mathbf{k}} f_{\mathbf{k}}^{\mathrm{eq}}(\mathbf{r}, t) \equiv \rho(\mathbf{r}, t) .
$$

Continuity adds a consistency condition for $u(\mathbf{r}, t)$ as, from Eq. (7), it further demands that

$$
\begin{aligned}
& \frac{\partial \rho}{\partial t}=\Omega^{-1} \sum_{\mathbf{k}}\left(-\frac{\partial f_{\mathbf{k}}^{\mathrm{eq}}}{\partial \mu}\right) \frac{\partial u}{\partial t}=-\frac{\partial u}{\partial t} \frac{\partial \rho}{\partial \mu} \equiv-\frac{\partial}{\partial \mathbf{r}} \cdot \mathbf{j} ; \text { so } \\
& \frac{\partial u}{\partial t}=\frac{\partial \mu}{\partial \rho} \Omega^{-1} \sum_{\mathbf{k}} \mathbf{v}_{\mathbf{k}} \cdot \frac{\partial}{\partial \mathbf{r}} f_{\mathbf{k}}(\mathbf{r}, t) .
\end{aligned}
$$

Equation (8) defines the local time dependence of the effective equilibrium, subject to the model's assumption that all relaxation events are instantaneous.

At global equilibrium we have $\mathbf{F}(\mathbf{r}) \equiv-\partial u / \partial \mathbf{r}$ and the Fermi-Dirac distribution of Eq. (6) exactly satisfies the stationary, collisionless QBE [26]. Away from equilibrium, the local structure of $f_{\mathbf{k}}^{\mathrm{eq}}(\mathbf{r}, t)$ forces the solution to be conserving. By a continuous and local resetting of the effective (though not the global) chemical potential $\mu-u(\mathbf{r}, t)$, the revised inelastic Drude Ansatz obeys the zero-trace requirement at every step of the model system's evolution. (Physically it also conforms to a thermodynamic rule of thumb: a disturbed system's eventual global relaxation tends first to pass rapidly to a temporary, locally defined equilibrium.)

With continuity now well controlled, the local equilibrium function forms the physical input to the otherwise purely formal solution of the full nonequilibrium distribution function. However, there is a cost. The procedure introduces a selfconsistent loop into the calculation since, through $\rho$ and therefore $u$, the reference equilibrium distribution is itself a functional of the actual distribution to be solved.

Here a further consideration appears: regardless of its inner detail, the QBE standing alone does not contain enough structure to close the physical transport problem. The computational loop requires a connection between the one-body nonequilibrium density $\rho$ and the locally induced force field $\mathbf{F}$. In the context of electron transport, closure comes through the constitutive relation that is the Poisson equation, tying $u$ to $\rho$ and generating the local induced electrostatic field, adjunct to the externally supplied driving field.

Conservation has to be taken seriously. If so, it is true that simplifying the collisional part of a transport problem is offset by the extra effort to obtain the auxiliary distribution. Nevertheless, as a practical matter, self-consistency cannot be avoided. For systems with nonuniform charge distributions (including almost every realistic mesoscopic case) kinetics and electrostatics become inextricable. A familiar example is the "pinch-off" phenomenon at the heart of a field-effect transistor's action.

\subsection{Kubo Formula}

Kubo [27, 29, 30] established a very general transport framework complementary to earlier kinetic methods. To appreciate the difference we recapitulate the goals of each perspective. 
Both in classical and quantum kinetics one starts from the abstract but complete multi-particle description in the system's phase space, and takes expectation values in the dynamical (Liouville) equation. Statistical averages are first performed over the general evolution, before any particular observable is identified for solution, and then subsets of the internal variables are further removed by systematic integration; a simple illustration is the reduction of Eq. (1) to (3).

The result is an enormous nesting of reduced but strongly coupled equations of motion for all the expectation values. To terminate the otherwise unmanageable hierarchy, higher-order many-body expectations must be factorized, in some physically guided way, and modelled as functionals of the lower-order ones. The classic instance of this is the "Stosszahlansatz" of Boltzmann: the stochastic argument leading to the characteristic shape of his collision terms.

As Section 2.1 shows, it is essential for any such averaging to preserve continuity, first and foremost at the single-carrier level (analogous higher-order identities, or sum rules [23], also exist). That this is a nontrivial matter is evident in the careful steps needed to make even the "simple" Drude model microscopically conserving.

By contrast, although in Kubo's method one also starts with the system's full quantum dynamical equation - given the Hamiltonian - the quantum commutation relations are first applied to it systematically. This has the effect of projecting the single-particle current operator, for example, uniquely into an equivalent, two-particle quantum correlation operator. Only after this mathematical procedure are physical averages taken on each side of the exact relation. This prescribes the specific relation between the mean, measurable current response and the mean (in principle also measurable) current-current correlation.

In summary, a kinetic equation works directly by functionally linking expectation values of particle correlations, operating at distinct orders in powers of the carrier density. This is possible only by first making a (conserving) approximation for the higher-level distributions in terms of the lower ones. For a Kubo formula, every such correlation stays on an equal footing. Expectations strictly come after the dynamical relationship between an observable and its next-higher-order analogue has been singled out. Ensemble averaging then projects both objects out of the original, complete Hamiltonian evolution.

We now consider the eigenstates of the Hamiltonian $H_{0}$ for a many-particle system of interest. An external time-dependent perturbation $H^{\prime}(t) \equiv \Theta(t) U(t) A$ begins to act at $t=0$ - hence the step function $\Theta(t)$ - such that an external field $U(t)$ couples to a system observable $A$. In charge transport, $A$ could be the density or the flux-density operator.

A single assumption is made in the Kubo analysis: the characteristic energy $\left\langle H^{\prime}(t)\right\rangle$ must be small enough (compared, say, to the free energy) to justify a finite, and in practice linear, perturbation expansion in powers of $H^{\prime}(t)$. For $t>0$ the perturbation preserves a one-to-one mapping between the basis states of $H_{0}$ and those of the complete Hamiltonian $H(t)=H_{0}+H^{\prime}(t)$. The dynamical states remain orthonormal but, to linear order, changes in their mutual orientations in Hilbert space (relative to the initial basis) can be shown to cancel exactly in the time-dependent expectation of any observable $B$ in the Heisenberg picture:

$$
\langle B(t)\rangle \equiv\left(\sum_{n} e^{-E_{n}(N) / k_{\mathrm{B}} T}\right)^{-1} \sum_{n}\left\langle\psi_{n}|B(t)| \psi_{n}\right\rangle e^{-E_{n}(N) / k_{\mathrm{B}} T},
$$


with

$$
B(t) \equiv \exp \left(\frac{i}{\hbar} \int_{0}^{t} H\left(t^{\prime}\right) d t^{\prime}\right) \cdot B \cdot \exp \left(-\frac{i}{\hbar} \int_{0}^{t} H\left(t^{\prime \prime}\right) d t^{\prime \prime}\right) .
$$

The trace in Eq. (9) runs over the equilibrium distribution for the whole $N$-particle system. Only those terms directly involving the time development of $B(t)$ contribute, since the expectations themselves are simply traces over the unperturbed eigenstates, populated at thermal equilibrium.

The convolution expressed in Eq. (10) simplifies on expanding its pair of unitary evolution operators to linear order to arrive at

$$
\begin{aligned}
B(t) \rightarrow & e^{i H_{0} t / \hbar} \cdot\left(1+\frac{i}{\hbar} \int_{0}^{t} e^{-i H_{0} t^{\prime} / \hbar} H^{\prime}\left(t^{\prime}\right) d t^{\prime} e^{i H_{0} t^{\prime} / \hbar}\right) \\
& \cdot B \cdot\left(1-\frac{i}{\hbar} \int_{0}^{t} e^{-i H_{0} t^{\prime} / \hbar} H^{\prime}\left(t^{\prime}\right) d t^{\prime} e^{i H_{0} t^{\prime} / \hbar}\right) \cdot e^{-i H_{0} t / \hbar} \\
\equiv & B_{0}(t)+\frac{i}{\hbar} e^{i H_{0} t / \hbar} \cdot \int_{0}^{t} d t^{\prime}\left(\widetilde{H}^{\prime}\left(t^{\prime}\right) \cdot B-B \cdot \widetilde{H}^{\prime}\left(t^{\prime}\right)\right) \cdot e^{-i H_{0} t / \hbar}
\end{aligned}
$$

where $B_{0}(t) \equiv e^{i H_{0} t / \hbar} \cdot B \cdot e^{-i H_{0} t / \hbar}, \quad \widetilde{H}^{\prime}(t) \equiv e^{-i H_{0} t / \hbar} \cdot H^{\prime}(t) \cdot e^{i H_{0} t / \hbar}$.

Expectations are taken on both sides. Since $\left\langle B_{0}(t)\right\rangle$ keeps its original equilibrium value (normally, this vanishes) we focus on the linear departure of the expectation

$$
\langle\delta B(t)\rangle \equiv\left\langle B(t)-B_{0}(t)\right\rangle .
$$

The cyclic trace property $\langle X \cdot Y\rangle=\langle Y \cdot X\rangle$ allows the exponentials in $\widetilde{H}^{\prime}(t)$ to be relocated within the right-hand side of Eq. (11) to yield the Kubo formula

$$
\langle\delta B(t)\rangle=\frac{i}{\hbar} \int_{0}^{t} d t^{\prime} U\left(t^{\prime}\right)\left\langle\left[A, B_{0}\left(t^{\prime}\right)\right]\right\rangle .
$$

The formula reveals a direct and deep equivalence between a one-body average response, such as current, on its left-hand side and a corresponding two-body correlation function within the integral on the right.

The significance of Eq. (12) is that it is universal for normal systems (those with a stable free-energy minimum). To make it concrete, consider a simple, circular one-dimensional (1D) metallic loop of length $L$, threaded by an even magnetic flux changing linearly in time. The flux induces an electric field $\mathcal{E} \Theta(t)$ everywhere on the perimeter. The electromagnetic Hamiltonian couples the vector potential $-\mathcal{E} t \Theta(t)$, unique up to a conservative gauge term, to the current operator $I(x)=q j(x)$ [31]. The expectation of the current itself responds according to

$$
\langle\delta I(x, t)\rangle=-i \frac{q^{2} \mathcal{E}}{\hbar} \int_{0}^{t} d t^{\prime} \int_{0}^{L} d x^{\prime}\left\langle\left[j_{0}\left(x^{\prime}, 0\right), t^{\prime} j_{0}\left(x, t^{\prime}\right)\right]\right\rangle .
$$

from which the system conductance can be defined (uniformity means that $\langle I(x, t)\rangle$ in Eq. (13) does not depend on $x$ ) as

$$
G(t)=\frac{\langle\delta I(t)\rangle}{\mathcal{E} L} \equiv \frac{q^{2}}{\hbar} \int_{0}^{t} d t^{\prime} t^{\prime} C\left(t^{\prime}\right) \quad \text { for }
$$




$$
C\left(t^{\prime}\right) \equiv-i \int_{0}^{L} \frac{d x}{L}\left\langle\left[j_{0}(x, 0), j_{0}\left(0, t^{\prime}\right)\right]\right\rangle
$$

Equation (14) is much more general that its restricted derivation above suggests. Moreover it carries special implications for one-dimensional mesoscopic transport. The time integral involving the correlator $C$ is dimensionless, and behaves in a way closely similar to the dimensionless transmission factor of the Landauer model for quantization of $G$ in one-dimensional ballistic conductors (see below). For further insight, we cite the Kubo analysis of Kamenev and Kohn [32] for closed driven mesoscopic circuits, including the dominant Coulomb-screening effects expected in realistic nonuniform cases.

Another perspective on Eq. (14) comes from its long-time limit. Empirically, a driven normal conductor will always reach steady state, usually very rapidly. Therefore $\int_{0}^{\infty} t C(t) d t$ should be finite; all transients will have fully contributed to the time integral.

There is a critical proviso. The Hamiltonian must include one or more scattering mechanisms of a many-body nature: carrier-phonon terms, or carrier-carrier, or both. If $H_{0}$ is strictly a single-particle object with no dynamical coupling to collective excitations, it is straightforward to show that Eq. (14) will not have a well-defined asymptote unless an infinitesimally damped, but nevertheless phenomenological, factor $e^{-\eta t}$ is adjoined to $C(t)$.

What does this mean? If $H_{0}$ does not explicitly include many-body contributions of some kind, the long-time behaviour of the product $\left\langle\left[j_{0}(x, 0), j_{0}\left(0, t^{\prime}\right)\right]\right\rangle$ cannot guarantee convergence to a well-defined steady state. Stabilising it with a formal ad hoc damping factor (in fact this is the Fermi Golden Rule in disguise) is tantamount to mimicking damping effects that could not otherwise emerge except by multi-particle excitations; compare the analysis given by Hershfield [33.

How does natural many-body damping appear in the Kubo formula? The time dependence of the current operator $j_{0}(0, t)$ leads to the spreading out, among more and more collective modes, of its self-overlap in the correlator. In fashionable parlance, the injected single-carrier states become progressively "entangled" with the far more abundant set of multi-particle states within the environment.

At the quantum level, therefore, propagation is not by single carriers but by entangled clusters. Furthermore this entanglement is as much kinematic (via Pauli exclusion and microscopic conservation) as it is dynamic (via interactions). It follows that many-body arguments are necessarily implicated and that any analysis ought not to pass them by.

The act of taking the trace average destroys the coherences that remain encoded (albeit inexorably entangled) in the commutator. The observable result is the actual decay of $C(t)$; and by far the most important idea to keep in mind for this mechanism, as it manifests in $C(t)$, is that it always represents the evolution of dynamically correlated particle-pair states and not of autonomous single-particle entities. This is how conservation is maintained, while the indispensable processes of energy loss are quantified in terms of the rate of energy redistribution from the relatively few externally excited modes to the exponentially more numerous multi-pair excitations of the system. In a real scenario, the injected energy which is first spread out among the collective modes will be further dispersed (dissipated) by passage to the even larger thermal environment.

For a system with effective mass $m^{*}$, dimensional analysis of the correlator shows 
that its integral scales as

$$
\int_{0}^{\infty} t C(t) d t=\frac{\hbar}{m^{*} L} n \tau
$$

in which $n=\langle\rho\rangle$ is the mean carrier density and $\tau$ is a time constant that characterizes the magnitude of the integral. Clearly, while $\tau$ is the outcome of generally very complex kinetic events deep in the system, it quantifies the observable attrition rate due to averaging over the "landscape" of all physically possible many-body excitations. The Kubo expression comes down to

$$
G=\frac{q^{2} n \tau}{m^{*} L}
$$

recognisable as the classic Drude formula [34.

A clarification is in order. It is sometimes claimed that the Kubo prescription is an artifice, a somewhat academic result restricted to idealized systems where global charge transfer poses no issue [4]. The implication is that, to push it into useful form, extra assumptions foreign to the spirit of the problem are necessary.

That is not so. Passing over the fact that practical working circuits are electrically closed by definition [35, the Kubo formula extends with equal rigour to any sub-system of a normal conductor (having an absolutely stable ground state), no matter whether the structure under study has closed or open boundary conditions, is macroscopic or mesoscopic, homogeneous or inhomogeneous.

It can be argued that Kubo's result is all the more powerful, applied to open structures with charge transfer to external reservoirs. This is because of the formula's inbuilt and particularly stringent constraints on global, over and above simply local, charge conservation. Interested readers are referred to the electrodynamic analyses by Magnus and Schoenmaker [36] and Sols [37] in addition to Ref. [32].

\subsection{Field-theoretical (Green-function) Methods}

The analysis of response and transport reaches its most complete form in many-body quantum kinetics, nowadays set in the formalism of quantum fields [23, 29, 30, 38, 39. This is not the place to give more than a verbal sketch but at least it can be said that semiclassical kinetics (post-Boltzmann), the Kubo formalism, and much more are subsumed in the field-theoretical point of view, commonly also referred to as the Green-function approach.

As the single-particle distribution $f_{\mathrm{k}}(\mathbf{r}, t)$ is the fundamental quantity of the QBE, so the one-body density matrix

$$
\begin{aligned}
\rho\left(\mathbf{r}, t ; \mathbf{r}^{\prime}, t^{\prime}\right) \equiv & -\Theta\left(t-t^{\prime}\right)\left\langle\psi(\mathbf{r}, t) \psi^{\dagger}\left(\mathbf{r}^{\prime}, t^{\prime}\right)\right\rangle \\
& +\Theta\left(t^{\prime}-t\right)\left\langle\psi^{\dagger}\left(\mathbf{r}^{\prime}, t^{\prime}\right) \psi(\mathbf{r}, t)\right\rangle
\end{aligned}
$$

becomes the fundamental one for the dynamical equations of quantum kinetic analysis. The expression above has the following interpretation. The Hilbert space of manyelectron states is now extended to arbitrary numbers of electrons, and the statistical ensemble average $\langle\cdots\rangle$ traces over all of them.

Acting on this greatly augmented state space, the operator $\psi^{\dagger}\left(\mathbf{r}^{\prime}, t^{\prime}\right)$ "creates" a particle at the space-time point $\left(\mathbf{r}^{\prime}, t^{\prime}\right)$ by projecting any $N$-particle state to another with $N+1$ particles, one of which is initially at $\mathbf{r}^{\prime}$. In the same vein its conjugate 
$\psi(\mathbf{r}, t)$ "annihilates" an electron at $(\mathbf{r}, t)$ and sends any $N$-electron state to some $N-1$ state with an electron initially absent at $\mathbf{r}$. The operators are cast in the Heisenberg picture, as with Eq. (10) above. Fermion antisymmetry means that, at equal times, they satisfy the anticommutation relation

$$
\psi(\mathbf{r}, t) \psi^{\dagger}\left(\mathbf{r}^{\prime}, t\right)+\psi^{\dagger}\left(\mathbf{r}^{\prime}, t\right) \psi(\mathbf{r}, t)=\delta\left(\mathbf{r}-\mathbf{r}^{\prime}\right)
$$

In Eq. (17), the time ordering reverses the role and sign of its fermion operator pair: for $t>t^{\prime}$ we add a single-electron excitation, later to be removed. This probes the overlap, or correlation, within the evolving one-body distribution and is the simplest measure (though theoretically insufficient for conductance) of the system's relaxation during the interval $t-t^{\prime}$. Conversely, for $t^{\prime}>t$ the operation removes an electron - adds a hole - and restores it later to measure relaxation of the hole in the interacting-electron background. (The choice of time ordering is for convenience since, mathematically, a hole that evolves forwards in time looks just like an electron evolving "backwards" in time.)

Given a system's interacting Hamiltonian, its many-body Schrödinger equation leads to a conjugate pair of coupled equations of motion for $\rho\left(\mathbf{r}, t ; \mathbf{r}^{\prime}, t^{\prime}\right)$. The objects $\psi$ and $\psi^{\dagger}$ act like quantum-field operators and $\rho$ has all the characteristics of the Green function, or "propagator", for the coupled equations. This makes the language highly abstract; in compensation it also makes accessible, to practical condensedmatter transport calculations, some sophisticated techniques of field theory such as Feynman-diagram analysis [29] and Keldysh time-evolution contours [39].

As a two-point double-time quantity, the complexity of the density matrix exceeds that of the solution to the QBE. On the other hand, it necessarily encodes far more information: its evolution admits every possible quantum coherence effect, at every scale. Nevertheless, despite the quantum subtleties, the way in which the evolutionary character of $\rho$ is interpreted stays conceptually quite close to the older understanding that motivates the Boltzmann equation.

The quantum many-body equations' kinematic complexity is only the beginning. The creation-annihilation pair within the density matrix couples to the Hamiltonian such that two-pair, three-pair and, in general, multi-pair excitations all enter and contribute to its space-time development. Their interaction within the density matrix appears as a hierarchy of convolutions which represent the full generalization of Boltzmann's collision terms: there is a "scattering-out" component, or self-energy, that removes particle strength from $\rho$ and a "scattering-in" component, or vertex, that tends to restore particle strength.

Self-energy and vertex parts are intricate functionals of the higher-order multi-pair excitations coupled to the original electron-hole pair $\left(\psi^{\dagger} \psi\right.$ or $\left.\psi \psi^{\dagger}\right)$ represented by $\rho$. To satisfy particle conservation it is not only imperative for both parts to be retained in any approximate treatment of the dynamics but that they should be inter-related in a highly specific way, through the Ward identities 30 . Strategies for securing the essential conserving properties within approximate models of quantum transport are fully explored by Kadanoff and Baym [38]. Exactly the same considerations apply to the problem when posed in the language of Keldysh 39 .

The Ward identities generalize the optical theorem of scattering [30], which asserts that the loss of energy and momentum from an initial freely propagating state must be made up by the total gain of all the other states in the Schrödinger problem. Since unitary scattering couples all such modes, their evolution must be solved jointly. In transport, such modes are physically occupied, so energy-momentum conservation 
becomes inherently a many-body problem. The Ward identities quantify this collective coupling subject to conservation, in a way similar to the optical theorem's requirement on the underlying basis states. But while knowledge of the single-particle modes is a prerequisite to set up the many-body transport solution, it can never supplant it.

If the intrinsic particle-particle interactions are strong and/or long-ranged, normal perturbation theory will not work. The literature describes countless ingenious ways to deal with the internal correlations. It has a long pedigree, it is vast and it is still growing. For this discussion, the general References [23, 29, 30, 38, 39] must suffice.

Although internal interactions may be strong, one can still use linear response for the conductance of a metallic electron system, if driven by a weak enough external field [40]. Under this condition, the Kubo formalism is replicated within the Green-function picture [30].

A completely quantum treatment of $\rho$ is needed whenever the range of its quantum correlations begins to be reached by the physical size of the system or by the time scale for effects of interest; in less extreme applications the full description can be simplified. For example, to study processes whose rates are slow compared to the inverse Fermi frequency we can restrict the analysis to the density-matrix form

$$
\phi\left(\mathbf{r}, \mathbf{r}^{\prime} ; t\right) \equiv \lim _{t^{\prime} \rightarrow t^{+}} \rho\left(\mathbf{r}, t ; \mathbf{r}^{\prime}, t^{\prime}\right)=\left\langle\psi^{\dagger}\left(\mathbf{r}^{\prime}, t\right) \psi(\mathbf{r}, t)\right\rangle .
$$

Electron correlations are regarded as instantaneous, while their spatial nonlocality, or off-diagonal structure, remains an essential feature. The overall time evolution of the system, analysed quasi-statically (relative to the electrons), recalls the BornOppenheimer decoupling of slow and fast motions within electronic systems. The dynamics of this limit has been studied in depth by Mahan 30 .

The equal-time matrix $\phi\left(\mathbf{r}, \mathbf{r}^{\prime} ; t\right)$ is governed by a so-called master equation, one version of which is the Lindblad equation [41]. Its form can be distilled from its original quantum dynamical setting, though not without additional assumptions extending the arguments of Uehling and Uhlenbeck to deal with the off-diagonal nature of the density matrix.

As a final step the quantum Boltzmann equation re-emerges on restricting attention to $\phi$ in the neighbourhood of its diagonal elements $\phi(\mathbf{R}, \mathbf{R} ; t)$. From $\phi$, Wigner's distribution function can be defined as

$$
f_{\mathbf{k}}(\mathbf{R}, t) \equiv \int d^{3} r e^{-i \mathbf{k} \cdot \mathbf{r}} \phi(\mathbf{R}+\mathbf{r}, \mathbf{R}-\mathbf{r}, t) .
$$

If $\phi$ decreases sharply away from the central co-ordinate $\mathbf{R}$, the Wigner function behaves as a localized slowly varying quantity. Any remnant off-diagonal nonlocality is taken up into the dependence on wavevector $\mathbf{k}$. The latter spans a background electronic band structure, defined over some cell $\Omega$ centred on $\mathbf{R}$, whose size is small on the typical scale of $|\mathbf{R}|$ yet still large compared to the Fermi wavelength of the electrons; if not, the band-structure picture would not make good physical sense. As derived by Kadanoff and Baym [38] (with some assumptions for the k-dependent self-energy and vertex structures destined to become the locally defined Boltzmann collision term) this limit of the Wigner function $f$ becomes the semiclassical fermion occupancy, obeying a reduced equation identical to the QBE.

Distinct from the long-wavelength recovery of semiclassical kinetics, the original Wigner distribution can be studied and solved as a fully quantum distribution in its own right, equivalent to the equal-time density matrix and with its master equation transcribed to the Fourier domain. Wigner-function methods form an extensive 
body of work, particularly in high-field problems where Kubo and linear-response approaches lose their immediate utility. An instructive reference to this different transport methodology is Frensley [42].

\subsection{Landauer Formula}

Anticipating the eventual creation of structures with feature sizes much below any bulk mean free path, Landauer [43] and subsequent developers of his approach [4, 5, 6] were motivated to understand conductance as a mesoscopic phenomenon with less emphasis on kinetic relaxation and more on a hydrodynamic (in the quantum limit, wave-like) concept of the interaction with scattering sources. The sources are treated not so much as mediators of dynamical many-body excitations but as modifiers of single-particle ballistic "flow" which remains, at all events, microscopically free and undisturbed.

The analogy shifts from the staccato motion of colliding billiard balls towards the smooth geometry of a working airfoil. One might say - with a bit of poetic licence that the Landauer approach to mesoscopics aims to be holistic while standard ones are bound to remain thoroughly reductionist. In stressing a continuum-like rather than particulate formal description of transport, one feature of this viewpoint is a more diffuse theoretical link between the average response of a system and fluctuations about the average.

Landauer characterized his own viewpoint in part as ascribing conceptual parity to the roles of current and voltage [43. Either should equally well be able to act as the causative agent of transport, with its partner representing the effect. This is radically different from the Kubo analysis, for example, where an external field has absolute meaning as the applied stimulus to conduction while the current is invariably the system's causal response. Causality turns out to be intimately tied to the notion of dissipative relaxation.

It may appear ironic that the Landauer formula applied to 1D conductance does not actually require any novel assumptions, as introduced originally by Landauer, to establish it. To show that it does not, we derive it straightforwardly from canonical transport theory. The proof has several stages.

- First: we invoke the Kubo formula, Eq. (14), for the current traversing an open one-dimensional mesoscopic channel [37; the working channel is a uniform wire smoothly embedded within a sub-system itself forming a segment of a macroscopically closed driven loop [32]. The sub-system encompasses the mesoscopic interface regions, since these are comparable in size to the channel's conductive core. Even if the core is ballistic, scattering in the boundaries is a real and dominant effect. Equation (15) therefore applies.

- Second: existence of a steady state implies that the time integral of the correlator in Eq. (15) is finite. Therefore the conductance takes the form of Eq. (16):

$$
G=\frac{q^{2} n}{m^{*} L} \frac{\tau_{\text {in }} \tau_{\mathrm{el}}}{\tau_{\text {in }}+\tau_{\mathrm{el}}}
$$

where, by Matthiessen's rule, the stochastically independent collision rates $\tau_{\text {in }}^{-1}$ and $\tau_{\mathrm{el}}^{-1}$ add together and lead to the composite collision time $\tau=\left(\tau_{\mathrm{in}}^{-1}+\tau_{\mathrm{el}}^{-1}\right)^{-1}$.

- Third: the sub-system's operative length $L$ must be determined by the physical environment of the open configuration for the core plus boundary regions. In a ballistic structure, the effective mean free paths are no longer uniquely set by the 
bulk material, but by the dynamics at the interfaces. This implies that $L$ becomes, in effect, the maximum of the mean free paths $\lambda_{\text {in }}$ and $\lambda_{\mathrm{el}}$, or any others that can be theoretically (and experimentally) distinguished over the span of the device. Suppose the mean speed of the carriers within the system is $v_{0}$. Then

so

$$
L=\max \left\{\lambda_{\text {in }}, \lambda_{\text {el }}\right\} \equiv v_{0} \max \left\{\tau_{\text {in }}, \tau_{\text {el }}\right\}
$$

$$
\begin{aligned}
G & =\frac{q^{2} n}{m^{*} L} \frac{\lambda_{\text {in }} \lambda_{\mathrm{el}}}{v_{0}\left(\lambda_{\mathrm{in}}+\lambda_{\mathrm{el}}\right)} \\
& =\frac{q^{2} n}{m^{*} v_{0}} \frac{\lambda_{\mathrm{in}} \lambda_{\mathrm{el}}}{\max \left\{\lambda_{\mathrm{in}}, \lambda_{\mathrm{el}}\right\}\left(\lambda_{\mathrm{in}}+\lambda_{\mathrm{el}}\right)} \\
& =\frac{q^{2} n}{m^{*} v_{0}}\left(\frac{1}{2}-\frac{\left|\lambda_{\mathrm{in}}-\lambda_{\mathrm{el}}\right|}{2\left(\lambda_{\mathrm{in}}+\lambda_{\mathrm{el}}\right)}\right) .
\end{aligned}
$$

- Fourth: in the degenerate limit, the characteristic speed of the carriers becomes the Fermi velocity; $v_{0}=v_{\mathrm{F}}$. At the same time the density is given by $n=2 k_{\mathrm{F}} / \pi$ where $k_{\mathrm{F}}$ is the Fermi wavevector, related to $v_{\mathrm{F}}$ through the momentum: $\hbar k_{\mathrm{F}}=$ $m^{*} v_{\mathrm{F}}$. Substitution for $n$ and $v_{0}$ in Eq. (19) gives

$$
G=G_{0}\left(1-\frac{\left|\lambda_{\text {in }}-\lambda_{\text {el }}\right|}{\lambda_{\text {in }}+\lambda_{\text {el }}}\right) ; \quad G_{0} \equiv \frac{q^{2}}{\pi \hbar} .
$$

The expression in parentheses on the right-hand side of Eq. (20) operates in every way identically to the transmission factor $\mathcal{T}$ in the standard form of the Landauer conductance,

$$
G=G_{0} \mathcal{T} \text { with } \quad 0 \leq \mathcal{T} \leq 1 .
$$

What is the experimental distinction between Eq. (20) derived directly from the standard quantum Kubo formula, and Eq. (21) obtained by rather different physical arguments [4,5]? There is no empirical distinction at the level of so-called "two-probe" current-voltage measurements (both quantities being read off the same pair of probe terminals).

However, the underlying differences in origin and interpretation may be substantial. Bringing these out could be a matter of some interest, calling for additional measurements designed around, say, well constructed "four-probe" configurations to obtain current and voltage reliably and independently across the inner core. Also necessary would be improved experimental control of inelastic, over against elastic, scattering effects.

Another central quantity for testing issues of principle is current noise, providing knowledge of the two-body correlator $C$; recall Eq. (14). Noise properties lie beyond the capacity of two- and four-probe methods, which test only the singlecarrier response; but high-quality nonequilibrium noise measurements are difficult to carry through. The basic implications of existing ballistic-noise experiments, analysed quantum kinetically, have been addressed elsewhere [44.

In the Landauer equation (21), the ideal scenario plays out when $G$ reaches the full conductance quantum $G_{0}$. This occurs if, and only if, $\mathcal{T}=1$. The channel is then considered to be perfectly transparent to the carriers.

The Kubo-derived Eq. (20) attains ideality if, and only if, the mean free paths are equally matched, both consequently being equal to the ballistic length of the structure 
as a whole: interfaces as well as interposed conductor. This does not entail "perfect" totally collisionless - transport since both inelastic and elastic scattering act unfettered at the scale of the overall structure.

It is because the mesoscopic Kubo description necessarily incorporates the interface physics into that of the "real" device, sandwiched between, that collision events retain their explicit and pre-eminent role even in the case of ideally quantized ballistic conductance. This differs from the Landauer description in that (a) elastic scattering is present and robust, and (b) the physics of dissipation does not devolve to the asymptotic equilibrium regions in the leads [4 but acts co-cooperatively with the elastic effects. In Kubo, dissipation is the inalienable signature of dynamics in the active, nonequilibrium region.

The boundaries are always with us. The mesoscopic boundaries are the physical loci of all scattering, elastic and inelastic together. Yet it is inelastic scattering alone that stabilizes the steady state for every transport problem, irrespective of the device scale. The deeper reason why that is so concerns us in the following Section.

\section{Dissipation and Conductance}

\subsection{Dissipation}

As long as a conducting structure is ohmic, it dissipates its externally supplied power at the usual rate

$$
P=I V=G V^{2} .
$$

A circuit in which such dissipation occurs is insensitive to the microscopic details of conduction; it is essential only that there exist a path - any path at all - for energy loss. Should it happen that $G=G_{0}=77.4809 \mu \mathrm{S}$, it makes no difference to Eq. (22) whether this conductance belongs to a common-stock resistor, or is measured for a uniquely hand-crafted and very costly ballistic research device.

The key question is straightforward. In terms of its energetics, what distinguishes the process of conduction in a conventional channel from a ballistic one? A crude answer might be: nothing.

Unsatisfactory as it seems, such an answer does not fundamentally require too much elaboration. The universality of electrical energy dissipation is plain; what is at times less obvious is how to resolve the apparent incompatibility between ideally ballistic transport on the one hand and, on the other hand, the absolute necessity for accelerated electrical charges to shed their energy gain inelastically.

In the previous Sec. 2.2 we intimated that the physical answer to the origin of energy dissipation rests in the correlation structure of the Kubo formula, and in Sec. 2.4 we made direct use of that relation to recover, in a standard way, quantization of $G$ in a one-dimensional metallic mesoscopic conductor. Commensurability of the nondissipative (elastic) scattering length and the dissipative (inelastic) length, and of each of them with the operational size of the ballistic conductor, is the essential element in the emergence of quantization within the Landauer formula, as a corollary to the general Kubo analysis.

Dissipation is thus a given in normal transport and the necessity for its presence is widely appreciated. Nevertheless discussion sometimes still surrounds not the reality of dissipation, but its actual centre of operation. Is energy loss an active process, immediately tied to the dynamics of the structure under test and thus demanding 
explicit modelling [28]? Or is it instead a remote and passive effect, buried deep within the equilibrium background and hence free of all need to represent it within the physics of a viable transport model [4]

Kubo's formula reveals the first of the alternatives above to be the one that faithfully reflects the physics of dissipation. Conditioned as it is by the many-body correlation properties, the Kubo formula demands that inelastic relaxation be present and accounted for quantitatively within the correlator. Were elastic scattering to be the only process allowed, the system could not relax; it would have no mechanism for shedding the energy pumped into its carrier population by the driving field. The system would become grossly disequilibrated and steady state would be impossible.

\subsection{Fluctuations}

A concrete example illustrates the drastic thermodynamic consequences if mesoscopic transport were exclusively determined by elastic (nondissipative) scattering. We preface the example by introducing an essential idea, the fluctuations in a mesoscopic conductor. To that end we recall the Kubo relation in its alternative form [29, 30] (we will specialize to $1 \mathrm{D})$,

$$
G=\frac{1}{k_{\mathrm{B}} T}\left\langle\mathcal{I}_{0} I_{0}(0)\right\rangle ; \quad \mathcal{I}_{0} \equiv \int_{0}^{\infty} d t I_{0}(t) .
$$

Equation (23) is the zero-frequency - that is, steady-state - version of the fluctuationdissipation theorem (FDT). It asserts that the low-field conductance is defined by the equilibrium fluctuations, or statistical self-correlation, of the current about its zero mean; the very heart of the Kubo formula. In Ziman's words [29]: "conductivity is a property inherent in the quantum-mechanical description of the unperturbed system; the application of a weak electric field merely exposes the time-correlations of the fluctuating components of the electric current in the equilibrium state." This is a general reformulation of Einstein's early conclusion that random (Brownian) motion determines the measurable mobility of a test particle.

The fluctuation-dissipation theorem's significance is that every microscopically conserving transport model must imply it. This covers every model that can be consistently formulated within quantum kinetics (Sec. 2.3) including, apart from the Kubo formula, the entire class of quantum Boltzmann theories (Sec. 2.1).

We emphasize that the FDT is a necessary consequence of conservation. A transport model, if forced to assume the fluctuation-dissipation relation as an additional external hypothesis, could not have incorporated the physically required level of conservation in the first place. As with the Drude model of Eq. (5) the remedy would be to restructure the model's equation of motion to make it microscopically conserving; the theorem would then follow. It is not enough simply to add the FDT phenomenologically, without reconsidering the model's underlying kinetics.

The scope of the FDT omits nonequilibrium fluctuations, however. While the latter must conform to the FDT in the linear weak-field limit, the relation by itself cannot reveal anything of the system's behaviour as soon as it departs from linear response. A genuinely nonlinear kinetic framework is needed.

To compare the wider consequences of active dissipation with those of a dissipation-free picture of ballistic conductance, we revisit a conserving BoltzmannDrude description of uniform 1D mesoscopic transport [45]. The model recovers the FDT in the weak-field regime and reproduces the Landauer conductance from Eq. 
(20). Away from that limit it gives a quantitative account of nonequilibrium current fluctuations for a ballistic conductor at high driving fields 44 and thus offers a testbed for such effects.

We return to the zero-frequency conductance given by that model for metallic (degenerate) carriers, but with a new working premise: the mesoscopic sample length $L$ is to be set independently of the scattering mechanisms at the sample boundaries (as just one instance, it might be visualized to be the wire's dimension in fabrication). Combining Eqs. (18) and (23), the low-field conductance is accompanied by the current-fluctuation strength

$$
S(0) \equiv\left\langle\mathcal{I}_{0} I_{0}(0)\right\rangle=k_{\mathrm{B}} T G_{0} \frac{2 \lambda_{\text {in }} \lambda_{\mathrm{el}}}{L\left(\lambda_{\text {in }}+\lambda_{\mathrm{el}}\right)}
$$

in the zero-voltage limit.

The same theory also yields the corresponding nonlinear current-current fluctuation at higher applied voltages. For finite $V$ this is [45]

$$
S(V)=S(0)\left[1+\left(\frac{q V}{m^{*} v_{\mathrm{F}}^{2}}\right)^{2}\left(\frac{\lambda_{\text {in }}}{L}\right)^{2}\left(2-\frac{\lambda_{\text {in }}^{2}}{\left(\lambda_{\text {in }}+\lambda_{\text {el }}\right)^{2}}\right)\right]
$$

while the power dissipation in the model remains as

$$
P=V^{2} G_{0} \frac{2 \lambda_{\text {in }} \lambda_{\mathrm{el}}}{L\left(\lambda_{\text {in }}+\lambda_{\mathrm{el}}\right)}
$$

so the energy loss rate stays directly proportional to $S(0)$.

The zero-field fluctuation strength $S(0)$ scales with the typical energy $k_{\mathrm{B}} T$ of a carrier at thermal equilibrium. What, then, does the difference of the current fluctuations, $S(V)-S(0)$, represent? It can be characterized in terms of an effective "hot-carrier" excess temperature $\Delta T \equiv T(S(V) / S(0)-1)$; that is, it measures the overhead of energy converted into - and retained as - the mean kinetic energy of highly excited carriers accelerated by the driving field.

Thus the nature of $S(V)-S(0)$ is nondissipative; excess fluctuations are not associated with the energy transfer that manifests as Joule heating in the structure and its surroundings, since this is keyed to $S(0)$. Rather, it reflects the average inventory of energy built up and stored in the carrier population by the underlying dynamical processes that sustain the nonequilibrium carriers in steady state.

Consider next what would happen in the limit of asymptotically weak inelastic scattering [46]; all along, $L$ stays fixed by hypothesis. Then $\lambda_{\text {in }} \gg L, \lambda_{\text {el }}$ and leads to

$$
\begin{aligned}
& S(0) \rightarrow k_{\mathrm{B}} T G_{0} \frac{2 \lambda_{\mathrm{el}}}{L}, \text { while } \\
& S(V) \rightarrow S(0)\left[1+\left(\frac{q V}{m^{*} v_{\mathrm{F}}^{2}}\right)^{2} \frac{\lambda_{\mathrm{in}}^{2}}{L^{2}}\right] \text { so } \\
& \Delta T \gg T .
\end{aligned}
$$

This example highlights the consequence of taking inelastic scattering completely out of the mesoscopic transport picture. Even though conductance is not qualitatively affected, the nonequilibrium current fluctuations diverge; they do so, in fact, for any nontrivial applied voltage. As a result, while the low-field current response $I=G V$ 
may well remain finite - in a strictly formal sense - its deviation from that average blows out uncontrollably. This makes the very possibility of measuring the current's mean value empirically void.

A mesoscopic calculation done strictly in linear response but excluding from consideration all inelastic collisions will, notwithstanding, yield a plausible value for the low-field conductance - and fail to reveal the uncontrollably wild behaviour of the nonequilibrium current fluctuations, which is inevitable except at $V=0$. Deprived of any mechanism for inelastic energy loss, a prediction that appears quite reasonable at linear response must, in reality, hide the seriously unphysical nature of its associated fluctuations.

One can contrast the nonequilibrium implications of the calculation for a sample length that is in fact physically delimited by the scattering physics in the interface regions, as described previously. With this understanding of $L$, and assuming now that the inelastic mean free path is largest one in the device configuration, we have $L=\lambda_{\text {in }} \gg \lambda_{\text {el }}$. Substitution into Eq. (24) shows that $S(0)$ and $G$ are diffusively dominated by elastic scattering. Meanwhile, the excess fluctuations, no longer divergent, are well behaved in all circumstances.

The example above entails four considerations:

(i) A description of transport in a normal, thermodynamically stable system should incorporate a specific model for inelastic scattering; otherwise, the existence of a meaningful steady state is not guaranteed. This constraint applies just as much to mesoscopic charge transport as to any other setting for electrical conduction. Inelasticity is intimately linked to the dissipative energetics of transport and is essential to the stability of the steady state.

(ii) In the mesoscopic context, the scales for both elastic and inelastic scattering become comparable to each other and to the operative length of a conductor. Conventional bulk notions of a purely geometric device "length"; that is, one conceptually divorced from the particular mechanisms for real scattering, are problematic when device dimensions shrink dramatically and its boundaries are wide open to direct energy exchange.

(iii) The current-fluctuation structure of a conductor is inseparable from the current response. Transport models that are conserving always lead to a fluctuationdissipation relation, which will automatically take care of the essential link between response and fluctuations.

(iv) Particularly in the meso- and nanoscopic regimes, strictly linear models of current response may not be sufficient to discriminate among subtle competing dynamical phenomena. For a fuller picture it would be necessary to develop both nonlinear theoretical descriptions and experiments that better revealed those properties lying beyond weak-field measurement [44].

\subsection{Many-body Aspects}

In strongly correlated systems, transport solutions that appear straightforward in the semiclassical regime must be re-examined in the appropriate many-body setting. Of the Green-function techniques, the Keldysh analysis [39] provides the paradigm of current interest [6]. The primary physical issues confronting a many-body approach remain (1) whether it is inherently conserving; and (2) whether the FDT is contained 
in it rather than having to be phenomenologically assumed. A consistent Keldyshbased analysis will automatically meet both requirements.

Since the first aim is to compute the single-particle current response, one has to look for the particulars by which strong multi-particle interactions filter down to the one-body level and modify the noninteracting form of the conductance. The Kubo formalism already identifies this nexus: it is the architecture of the correlation function $\langle j(t) j(0)\rangle$. In the Keldysh analysis, it is the self-energy associated with the one-body propagator (Green function) that ties the inner details of the many-body correlations to the outward behaviour of the current response.

Briefly stated, a correlated system at rest possesses a self-energy structure precisely attuned to the scattering amplitude (interaction vertex) between carrier pairs. This tuning is essential for microscopic conservation. It is encoded in the Ward identities [30].

Scattering effects that appear in the form of a nonequilibrium correction to the one-body self-energy (the so-called energy relaxation rate) are systematically offset by counter-terms that are nonequilibrium vertex parts, which are not of single-particle form. However, there is a natural quantity that accommodates both types: the twoparticle propagator or correlation function, characteristic of the Kubo formula. In the semiclassical limit, the fundamental cancellations between self-energy and vertex terms manifest as the principle of detailed balance and determine the conserving structure of the Boltzmann collision integral.

The Keldysh formalism lets one track the modified self-energy structure in a controlled way. It can then be compared with the equilibrium value. Taken in isolation, the self-energy difference defines the loss rate in one-body propagation: the apparent exponential attenuation in the strength of a single injected carrier reflecting its progressive entanglement with innumerable many-body excitations.

Even though the computational accent is on the self-energy we stress that, within the Keldysh expansion, the Ward identities apply and require those contributions, not expressible as one-body self-energy parts (namely the vertex corrections) to appear explicitly in the overall relaxation. They do so on fully equal terms with changes that keep the formal shape of a self-energy. The complete form of the correlator $\langle j(t) j(0)\rangle$, and thus (via Kubo and the FDT) the conductance, is the sum of two parts: selfenergy and vertex terms. Together, and only together, they determine the momentum relaxation rate [30, whose inverse appears as $\tau$ in the Kubo formula, Eq. (15).

A well-known application of Keldysh analysis is the Meir-Wingreen formula [6]

$$
G=-G_{0} \int d E \operatorname{Im}\left\{\left\langle\Gamma \mathcal{G}^{r}\right\rangle\right\} \frac{\partial}{\partial \mu} f^{\mathrm{eq}}(E-\mu)
$$

where the energy integral is over the asymptotic carrier band of the (noninteracting) current reservoirs attached to the central (interacting) system, and the expression whose imaginary part is taken represents the trace over the internal many-body excitations of the core. The Green function $\mathcal{G}^{r}$ is in Keldysh retarded form and $\Gamma$ encodes the inner core's coupling to the leads.

In the noninteracting case, $\Gamma(E, \omega)=2 \pi \mathcal{T}(\omega) \delta(E-\omega)$ is the one-body transitionmatrix operator [29] for the device treated as a barrier to single-particle propagation. The corresponding free single-particle propagator is $\mathcal{G}^{r}\left(\omega, \omega^{\prime}\right)=1 /\left(\omega-\omega^{\prime}+i 0^{+}\right)$and the Meir-Wingreen kernel goes to 


$$
\begin{aligned}
\Gamma \mathcal{G}^{r} & =\int \frac{d \omega^{\prime}}{2 \pi} \Gamma\left(E, \omega^{\prime}\right) \int \frac{d \omega}{2 \pi} \frac{e^{-i \omega \eta}}{\omega-\omega^{\prime}+i 0^{+}} \\
& =-i \mathcal{T}(E) ; \quad \eta \rightarrow 0^{+} .
\end{aligned}
$$

Note again the need for an asymptotic convergence factor to force this nondissipative analytic structure to simulate a stable steady state, as explained in the preceding section. If $\langle\mathcal{T}(E)\rangle$ varies slowly with energy, Eq. (25) recovers the Landauer formula Eq. (21). In the interacting case, the integral on the right side of Eq. (25) both formalizes and extends Landauer's phenomenological transmission coefficient.

For a system thus described - noninteracting or even interacting - the essential functions of dissipation and inelasticity appear to lack clear definition in Eq. (25). In that respect, three basic many-body aspects still await validation within its expression.

- Relation to Kubo formula and FDT: how is the canonical form of the two-particle Kubo correlation function recovered from the integrand on the right-hand side of Eq. (25)?

- Tracking the conserving structure of $\Gamma$ : how does it conform to the Ward identities, dynamically interconnecting the self-energy and vertex components?

- Auditing the mechanisms of energy loss: how do $\Gamma$ and $\mathcal{T}$ encode energy transfer (inelasticity) from the driving field to the environment, via many-body interaction(s)?

\section{Summary}

Mesoscopic electrical transport is now a major discipline within condensed matter, and has been so for almost three decades. Yet more than ever it is a rapidly changing, intellectually stimulating and economically vital research area. In large part, its theoretical initiatives are still fed by the astonishing growth, in sophistication and versatility, of materials technology.

For all of those reasons it is important to hold to the fore, with clarity, the conceptual underpinnings of transport theory. In mesoscopics its points of reference are, and will remain, precisely those that have governed the general understanding of quantum kinetics: chiefly, the conservation laws and the close interplay of electromagnetism with thermodynamics, which leads to the universality of energy dissipation in real mesoscopic systems.

The maturity of kinetic theory has been arrived at by a collective effort going on, without pause, since the formulation of quantum mechanics. Its legacy to mesoscopics is a fruitful, almost extravagant arsenal of well crafted and tested approaches to solving problems. This applies not just to nonequilibrium mesoscopics but also to the next step down, the quasi-atomic scale.

We have revisited the main, and already classic, kinetic techniques with the goal of teasing out their common unifying strands. These relate directly to the issues that will always confront mesoscopic transport theory: microscopic conservation and, most particularly, the accounting of energy dissipation at small scales. If a theory wants to replicate their explicit consequences, especially in a novel setting, it should first be able to demonstrate that it explicitly respects their agency.

The dissipation issue led us to elucidate how the nonlinear properties of energy loss processes hold the key to establishing thermodynamic stability within 
a mesoscopic transport description. The task of detailing in full the physics of dissipation outranks even those pragmatic and increasingly pressing questions now driving theoretical initiatives towards high fields.

Far from remote icons or symbolic figures for lip service, the unifying ideas that we have traced here are the immediate and prime constraints - "reality checks" on every mesoscopic model aspiring to serve the novel demands of device design by embodying, as practically and flexibly as possible, deeply established physical rules. We hope that this paper may help both as a reminder of them and as a pointer to the questions that need to be addressed by mesoscopic transport theories, before any answers are ventured.

\section{Acknowledgment}

One of us (MPD) acknowledges the support of the Asia-Pacific Centre for Theoretical Physics, Pohang, South Korea, where this work was presented as part of the Focus Programme of September 2011. In particular he thanks Professors Peter Fulde and Jongbae Hong for their hospitality.

\section{References}

[1] P. A. Lee and A. D. Stone, Phys. Rev. Lett 55, 1622 1985).

[2] B. J. van Wees et al., Phys. Rev. Lett. 60, 848 (1988).

[3] D. A. Wharam et al., J. Phys. C: Solid State Phys. 21, L209 (1988).

[4] Y. Imry and R. Landauer, Rev. Mod. Phys 71, S306 (1999).

[5] Ya. Blanter and M. Büttiker, Phys. Rept. 336, 1 (2000).

[6] Y. Meir and N. S. Wingreen, Phys. Rev. Lett. 68, 2512 (1992); A.-P. Jauho, N. S. Wingreen and Y. Meir, Phys. Rev. B 50, 5528 (1994).

[7] Y. Imry, Introduction to Mesoscopic Physics (2nd edn, Oxford University Press, Oxford, 2008).

[8] L. L. Sohn and L. P. Kouwenhoven, Mesoscopic Electron Transport (Springer, Berlin, 1997).

[9] T. Ihn, Electronic Quantum Transport in Mesoscopic Semiconductor Systems (Springer, Berlin, 2004).

[10] T. Ouisse, Electron Transport in Nanostructures and Mesoscopic Devices (Wiley, London, 2008).

[11] Y. V. Nazarov and Y. M. Blanter, Quantum Transport: Introduction to Nanoscience (Cambridge University Press, Cambridge, 2009).

[12] M. Di Ventra Electrical Transport in Nanoscale Systems (Oxford University Press, Oxford, 2008).

[13] E. Ackermans and G. Montambaux, Mesoscopic Physics of Electrons and Photons (Cambridge University Press, Cambridge, 2007).

[14] P. A. Mello and N. Kumar, Quantum Transport in Mesoscopic Systems: Complexity and Statistical Fluctuations (Oxford University Press, Oxford, 2004).

[15] Y. Murayama Mesoscopic Systems: Fundamentals and Applications (Wiley-VC, Berlin, 2001).

[16] K. Richter, Semiclassical Theory of Mesoscopic Quantum Systems (Springer, Berlin, 2000).

$[17]$ D. K. Ferry and S. M. Goodnick, Transport in Nanostructures (Cambridge University Press, Cambridge, 1999).

[18] S. Datta, Electron Transport in Mesoscopic Systems (Cambridge University Press, Cambridge, 1997).

[19] E. A. Uehling and G. E. Uhlenbeck, Phys. Rev. 43, 552 (1933).

[20] D. N. Zubarev, V. Morozov and G. Röpke, Statistical Mechanics of Nonequilibrium Processes: Basic Concepts, Kinetic Theory (John Wiley \& Sons, New York, 1996).

[21] J. M. Ziman, Principles of the Theory of Solids (Cambridge University Press, Cambridge, 1971), Ch 7.

[22] C. E. Korman and I. D. Mayergoyz, Phys. Rev. B 54, 17620 (1996).

[23] D. Pines and P. Nozières, The Theory of Quantum Liquids I (Benjamin, New York, 1966).

[24] M. P. Greene, H. J. Lee, J. J. Quinn, and S. Rodriguez, Phys. Rev. 177, 1019 (1969).

[25] N. D. Mermin, Phys. Rev. B 1, 2363 (1970). 
[26] This is a generic requirement on $f^{\mathrm{eq}}$ and brings in the principle of detailed balance for Eq. (4). For all state labels $\mathbf{k}, \mathbf{k}^{\prime}$, the identity holds. Equivalently,

$$
f_{\mathbf{k}}^{\text {eq }} Q_{\mathbf{k}^{\prime} \leftarrow \mathbf{k}}\left(1-f_{\mathbf{k}^{\prime}}^{\text {eq }}\right) \equiv\left(1-f_{\mathbf{k}}^{\text {eq }}\right) Q_{\mathbf{k} \leftarrow \mathbf{k}^{\prime}} f_{\mathbf{k}^{\prime}}^{\text {eq }}
$$

$$
Q_{\mathbf{k} \leftarrow \mathbf{k}^{\prime}}=e^{-\left(E_{\mathbf{k}}-E_{\mathbf{k}^{\prime}}\right) / k_{\mathrm{B}} T} Q_{\mathbf{k}^{\prime} \leftarrow \mathbf{k}} .
$$

The requirement of detailed equilibrium between individual collision contributions reveals that the Boltzmann rates involve more than simply knowing the set of elementary scattering crosssections. Their explicit dependence on the thermal energy tells us that, unless the scattering is strictly elastic, many-body interactions in the system - at minimum, those coupling to the huge numbers of excitable modes in the heat bath - must enter at the microscopic level. It is a deeper manifestation of the same physics that underlies Brownian motion, intimately associated with both fluctuations and dissipation.

[27] R. Kubo, J. Phys. Soc. Jpn. 12. 570 (1957).

[28] M. P. Das and F.Green, J. Phys: Condens. Matter 21, 101001 (2009).

[29] J. M. Ziman, Elements of Advanced Quantum Theory (Cambridge University Press, Cambridge, 1969).

[30] G. D. Mahan, Many-Particle Physics (Plenum, New York, 1981).

[31] where

$$
j(x)=-\frac{i \hbar}{2 m^{*}} \lim _{x^{\prime \prime} \rightarrow x}\left\{\left(\frac{\partial}{\partial x}-\frac{\partial}{\partial x^{\prime \prime}}\right)\left|x^{\prime \prime}\right\rangle\langle x|\right\}
$$

for a system with a quadratic energy dispersion and effective mass $m^{*}$.

[32] A. Kamenev and W. Kohn, Phys. Rev. B 63, 155304 (2000).

[33] S. Hershfield, Phys. Rev. Lett. 70, 2134 (1993).

[34] M. P. Das and F. Green, J. Phys: Condens. Matter 15, L687 (2003).

[35] The reason goes back to the Maxwell equations, in which electromagnetic fields and currents are topologically interlinked. Physically consistent distributions of carrier flux $\mathbf{j}(\mathbf{r})$ over a region of space have nonvanishing vector-circulation fields $\nabla \times \mathbf{j}(\mathbf{r})$, so the spatial domain of the flux must be multiply connected. The transport physics in such a globally determined, multiply connected but closed topology [36] is not replicable as a passive transfer of carriers from higher to lower (conservative) potentials. Around a closed circuit there is no "higher" or "lower" 32 and the external field acting within it is nonconservative [36] 37.,

[36] W. Magnus and W. Schoenmaker, Quantum Transport in Submicron Devices (Springer, Berlin, 2002).

[37] F. Sols, Phys. Rev. Lett. 67, 2874 (1991).

[38] L. P. Kadanoff and G. Baym, Quantum Statistical Mechanics (Benjamin, New York, 1962).

[39] D. C. Langreth, in Linear and Nonlinear Electron Transport in Solids, J. T. Devreese and V. E. van Doren eds (NATO Advanced Studies Series, Plenum, New York, 1976), pp 3-32.

[40] Again, this is true provided the system is normal and stable; phenomena such as superconductivity and the fractional quantum Hall effect require special measures to address the nature of their ground state, before obtaining a description of the response, which always involves excitations out of the ground state.

[41] A. K. Rajagopal, Phys. Lett. A 246, 237 (1998).

[42] W. R. Frensley, Rev. Mod. Phys. 62, 745 (1990); ibid. 63, 215 (1990).

[43] R. D. Landauer, IBM J. Res. Dev. 1, 223 (1957), reprinted (with comment) as J. Math. Phys 37, 5259 (1996).

[44] F. Green, J. Thakur, and M. P. Das, Phys. Rev. Lett. 92, 156804 (2004).

[45] F. Green and M. P. Das, Fluct. Noise Lett. 1, C21 (2004).

[46] This is equivalent to attaching the additional asymptotic convergence factor $e^{-\eta t}$ to the Kubo correlation function $C(t)$, securing a stable limit within purely single-particle Hamiltonian models; recall the discussion following Eq. (14). 